\title{
QUADRATIC 0-1 PROGRAMMING: TIGHTENING LINEAR OR QUADRATIC CONVEX REFORMULATION BY USE OF RELAXATIONS
}

\author{
Alain Billionnet $^{1}$, Sourour Elloumi ${ }^{2}$ And \\ Marie-Christine Plateau ${ }^{2}$
}

\begin{abstract}
Many combinatorial optimization problems can be formulated as the minimization of a $0-1$ quadratic function subject to linear constraints. In this paper, we are interested in the exact solution of this problem through a two-phase general scheme. The first phase consists in reformulating the initial problem either into a compact mixed integer linear program or into a $0-1$ quadratic convex program. The second phase simply consists in submitting the reformulated problem to a standard solver. The efficiency of this scheme strongly depends on the quality of the reformulation obtained in phase 1 . We show that a good compact linear reformulation can be obtained by solving a continuous linear relaxation of the initial problem. We also show that a good quadratic convex reformulation can be obtained by solving a semidefinite relaxation. In both cases, the obtained reformulation profits from the quality of the underlying relaxation. Hence, the proposed scheme gets around, in a sense, the difficulty to incorporate these costly relaxations in a branch-and-bound algorithm.
\end{abstract}

Received January 1, 2006. Accepted November 28, 2007.

1 Laboratoire CEDRIC, ENSIIE, 18 allée Jean Rostand, 91025 Evry, France; billionnet@ensiie.fr

2 Laboratoire CEDRIC, Conservatoire National des Arts et Métiers, 292 rue Saint Martin,

75141 Paris, France; [elloumi;mc.plateau]@cnam.fr 
Résumé. Le problème de la minimisation d'une fonction quadratique en variables $0-1$ sous contraintes linéaires permet de modéliser de nombreux problèmes d'Optimisation Combinatoire. Nous nous intéressons à sa résolution exacte par un schéma général en deux phases. La première phase permet de reformuler le problème de départ soit en un programme linéaire compact en variables mixtes soit en un programme quadratique convexe en variables $0-1$. La deuxième phase consiste simplement à soumettre le problème reformulé à un solveur standard. L'efficacité de ce schéma est étroitement liée à la qualité de la reformulation obtenue à la fin de la phase 1. Nous montrons qu'une bonne reformulation linéaire compacte peut être obtenue par la résolution d'une relaxation linéaire. De même, une bonne reformulation quadratique convexe peut être obtenue par une relaxation semi-définie positive. Dans les deux cas, la reformulation obtenue tire profit de la qualité de la relaxation sur laquelle elle se base. Ainsi, le schéma proposé contourne, d'une certaine façon, la difficulté d'intégrer des relaxations, coûteuses en temps de calcul, dans un algorithme de branch-and-bound.

Keywords. Combinatorial optimization, quadratic 0-1 programming, linear reformulation, quadratic convex reformulation.

Mathematics Subject Classification. 90C10, 90C11, 90C20.

\section{INTRODUCTION}

Consider the following linearly-constrained zero-one quadratic program :

$$
Q 01: \operatorname{Min}\left\{F(x)=\sum_{i=1}^{n} q_{i} x_{i}+\sum_{i=1}^{n} \sum_{j=1, j \neq i}^{n} q_{i j} x_{i} x_{j}: x \in X\right\}
$$

where $X=\left\{x: \sum_{i=1}^{n} a_{k i} x_{i}=b_{k}, k=1, . ., m ; \sum_{i=1}^{n} a_{\ell i}^{\prime} x_{i} \leq b_{\ell}^{\prime}, l=1, . ., p ; x \in\{0,1\}^{n}\right\}$ is the feasible solution set of $Q 01$ and $q_{i}, q_{i j}, a_{k i}, b_{k}, a_{\ell i}^{\prime}, b_{\ell}^{\prime}$ are real numbers. Without loss of generality, we can assume that $q_{i j}=q_{j i}$. We denote by $\bar{X}$ the continuous-relaxation solution set of $Q 01$. Set $\bar{X}$ is obtained from $X$ by replacing $x \in\{0,1\}$ with $x \in[0,1]$.

Problem $Q 01$ is NP-hard [13]. It allows to formulate many Combinatorial Optimization problems such as graph bipartition, quadratic knapsack, and quadratic assignment. It also has several applications such as task allocation and capital budgeting. Due to its complexity, many heuristic solution methods have been proposed for it. For example, $[4,15,21,23]$ apply tabu search for the unconstrained 
problem, and local search heuristics are applied to graph bipartition problems in $[19,20]$.

Exact solution methods have also been proposed for solving Q01. In this paper, we focus on two main approaches: linear reformulation and quadratic convex reformulation. Linear reformulation techniques transform $Q 01$ into a mixed integer linear program. The most frequently used linearization was first introduced by Fortet in $[11,12]$ and is sometimes called the classical linearization. It consists in replacing each product $x_{i} x_{j}$ by a new variable $y_{i j}$ and adding a set of linear constraints that force $y_{i j}$ to be equal to $x_{i} x_{j}$, i.e. $y_{i j} \leq x_{i}, y_{i j} \geq x_{i}+x_{j}-1, y_{i j} \geq 0$, and $y_{i j}=y_{j i}$ for all $i \neq j$. A strengthening of the classical linearization by a family of valid inequalities was later proposed by Sherali et al. in [25,26]. They developed a Reformulation and Linearization Technique (RLT). Using quite different ideas, Glover [14] introduced an alternative linearization strategy that requires a much smaller set of additional variables and constraints than the classical linearization and RLT. This is why it is often called a compact linearization. Variants of this compact linearization were further used by several authors $[1,8,16]$.

Another class of exact solution methods aims at reformulating the objective function of $Q 01$ by a quadratic convex function. Doing this, solving the continuous relaxation of the reformulated problem becomes tractable in polynomial time. For example, Hammer and Rubin [17] devise a simple convexification method based on a smallest eigenvalue computation. Later, Carter [9] then Billionnet and Elloumi [5], Plateau et al. [24], and Billionnet et al. [6] study several families of quadratic convex reformulations and provide theoretical and computational comparisons between these families. All the reformulations proposed in $[5,6,24]$ are based on an exact solution of a quadratic semidefinite program. Among these reformulations, the one proposed in [6] and called QCR gives the provably best bound by continuous relaxation.

In this paper, we introduce a positive compact linearization method and we recall the quadratic convex reformulation method QCR [6]. We will focus on the fact that each of these methods takes profit from a preprocessing phase. This phase aims both at finding a suitable reformulation of the problem and at making the further resolution process as efficient as possible. To achieve this last objective, we use the common criterion that prefers reformulations yielding bounds that are as tight as possible by continuous relaxation. We will show how we build a positive linear compact reformulation once an RLT relaxation is solved and how it captures the bound obtained by this relaxation. In a similar way, QCR is built once an appropriate SDP relaxation is solved and it captures the bound obtained by SDP relaxation.

The rest of the paper is organized as follows: Linear compact reformulations and convex quadratic reformulations are presented in Section 1. In Section 2, we show how to build a positive compact linearization from the RLT solution. In Section 3, we show how to build a QCR reformulation from the solution of an SDP relaxation. Section 4 is a conclusion. 
Example. All along this paper, we will use the same example for illustration purposes: we consider the following 0-1 quadratic program $E$, which optimal value is -65 :

$$
\begin{aligned}
E: \operatorname{Min} & \phi(x)=-9 x_{1}-7 x_{2}+2 x_{3}+23 x_{4}+12 x_{5}-48 x_{1} x_{2}+4 x_{1} x_{3}+36 x_{1} x_{4} \\
& -24 x_{1} x_{5}-7 x_{2} x_{3}+36 x_{2} x_{4}-84 x_{2} x_{5}+40 x_{3} x_{4}+4 x_{3} x_{5}-88 x_{4} x_{5} \\
\text { s.t. } & \\
& x_{1}-2 x_{2}+5 x_{3}+2 x_{4}-2 x_{5} \geq 2 \\
& x_{1}+x_{2}+x_{4}+x_{5}=2 \\
& x_{1}, x_{2}, x_{3}, x_{4}, x_{5} \in\{0,1\} .
\end{aligned}
$$

\section{ReFormulation OF QUADRATIC 0-1 PROGRAMS}

In the context of this paper, a reformulation of $Q 01$ is any equivalent mathematical program $P$ with integer or mixed integer variables, that is either linear or quadratic convex. These equivalent reformulations are expected to preserve the feasible solution domain and the optimal value. Among all the possible reformulations, we choose two reformulation schemes that either use the same variables and constraints as program $Q 01$ or require a small number of additional variables and constraints. Let us first recall the linear compact reformulation of Glover.

\subsection{The compact linearization of Glover [14]}

The linear reformulation method proposed in [14] aims to replace non-linear expressions of $Q 01$ with a set of continuous variables. More precisely, take $z_{j}=$ $x_{j} \sum_{i=1, i \neq j}^{n} q_{i j} x_{i}$ and reformulate $Q 01$ by:

$$
\begin{aligned}
& R L_{g}: \operatorname{Min} F(x)=\sum_{i=1}^{n} q_{i} x_{i}+\sum_{j=1}^{n} z_{j} \\
& \text { s.t. } \\
& \sum_{i=1}^{n} a_{k i} x_{i}=b_{k} \quad k=1, \ldots, m \\
& \sum_{i=1}^{n} a_{\ell i}^{\prime} x_{i} \leq b_{\ell}^{\prime} \quad \ell=1, \ldots, p \\
& L_{j} x_{j} \leq z_{j} \leq U_{j} x_{j} \quad j=1, \ldots, n \\
& \sum_{i=1, i \neq j}^{n} q_{i j} x_{i}-U_{j}\left(1-x_{j}\right) \leq z_{j} \quad j=1, \ldots, n \\
& z_{j} \leq \sum_{i=1, i \neq j}^{n} q_{i j} x_{i}-L_{j}\left(1-x_{j}\right) \quad j=1, \ldots, n \\
& x \in\{0,1\}^{n}, z \in \mathbb{R}^{n}
\end{aligned}
$$


where $L_{j}$ and $U_{j}$ are respectively lower and upper bounds of the linear functions $\sum_{i=1, i \neq j}^{n} q_{i j} x_{i}$, computed as : $L_{j}=\operatorname{Min}\left\{\sum_{i=1, i \neq j}^{n} q_{i j} x_{i}: x \in X\right\}$ and $U_{j}=$ $\operatorname{Max}\left\{\sum_{i=1, i \neq j}^{n} q_{i j} x_{i}: x \in X\right\}$.

By optimality considerations, since coefficients of the $z_{j}$ variables in the objective function are positive, inequalities $z_{j} \leq U_{j} x_{j}$ and $z_{j} \leq \sum_{i=1, i \neq j}^{n} q_{i j} x_{i}-L_{j}\left(1-x_{j}\right)$ can be discarded.

In the following, we present a family of compact linearizations that are inspired from Glover's linearization. They are built from a first reformulation of the objective function as a constant plus a nonnegative function over $\bar{X}$, as described below.

\subsection{Positive linear COMPACT ReFormulation}

Suppose one has identified a constant $c$ and functions $L(x), f_{i}(x)$, and $g_{i}(x)$ that are nonnegative on the relaxed domain $\bar{X}$, and that satisfy:

$$
\forall x \in X, \quad F(x)=c+L(x)+\sum_{i=1}^{n} x_{i} f_{i}(x)+\sum_{i=1}^{n}\left(1-x_{i}\right) g_{i}(x) .
$$

This first reformulation of $F(x)$ is always possible since a quadratic pseudoboolean function can be written as a constant plus a quadratic posiform. Function $\sum_{k=1}^{K} C_{k} T_{k}$ is a quadratic posiform if all coefficients $C_{k}$ are positive and every $T_{k}$ is a literal or the product of two literals. A literal is either a variable $x_{i}$ or its complement $\left(1-x_{i}\right)$. Writing a quadratic pseudoboolean function as a constant, as large as possible, plus a posiform has addressed much interest in literature and we know it can be done by at least three different methods ([18] among others). For our example $E$, the largest constant equals -160 and we can write $\phi(x)=-160+41\left(1-x_{1}\right)+42\left(1-x_{2}\right)+x_{1}\left[48\left(1-x_{2}\right)+24\left(1-x_{5}\right)\right]+71 x_{2}\left(1-x_{5}\right)+x_{3}\left(40 x_{4}+3 x_{5}\right)+$ $x_{4}\left[7 x_{5}+95\left(1-x_{5}\right)\right]+\left(1-x_{1}\right)\left[4\left(1-x_{3}\right)+36\left(1-x_{4}\right)\right]+\left(1-x_{2}\right)\left[7 x_{3}+36\left(1-x_{4}\right)+13 x_{5}\right]+(1-$ $\left.x_{3}\right)\left(1-x_{5}\right)$.

From the first reformulation (1), we can now build our positive compact linearization $R L_{\text {comp }}$. For $i=1, \ldots, n$, we need an upper bound $\bar{f}_{i}$ (resp. $\bar{g}_{i}$ ) for function $f_{i}(x)$ (resp. $g_{i}(x)$ ) over the feasible solution set $X$. Let $R L_{\text {comp }}$ be the 
following mixed 0-1 linear program:

$$
\begin{aligned}
& R L_{\text {comp }}: \text { Min } F_{L}(x)=c+L(x)+\sum_{i=1}^{n} h_{i}+\sum_{i=1}^{n} h_{i}^{\prime} \\
& \text { s.t. } \\
& \sum_{i=1}^{n} a_{k i} x_{i}=b_{k} \quad k=1, \ldots, m \\
& \sum_{i=1}^{n} a_{\ell i}^{\prime} x_{i} \leq b_{\ell}^{\prime} \quad \ell=1, \ldots, p \\
& h_{i} \geq f_{i}(x)-\bar{f}_{i}\left(1-x_{i}\right) \quad i=1, \ldots, n \\
& h_{i}^{\prime} \geq g_{i}(x)-\bar{g}_{i} x_{i} \quad i=1, \ldots, n \\
& x \in\{0,1\}^{n} \\
& h_{i} \geq 0 \quad h_{i}^{\prime} \geq 0 \quad i=1, \ldots, n .
\end{aligned}
$$

The inequalities on $h_{i}$ and $h_{i}^{\prime}$ impose that, for any optimal solution $\left(x, h, h^{\prime}\right)$ for $R L_{\text {comp }}$, if $x_{i}=0$ then $h_{i}=0$ and $h_{i}^{\prime}=g_{i}(x)$; if $x_{i}=1$ then $h_{i}=f_{i}(x)$ and $h_{i}^{\prime}=0$. Hence, variable $h_{i}\left(\right.$ resp. $\left.h_{i}^{\prime}\right)$ is equal to $x_{i} f_{i}(x)\left(\operatorname{resp} .\left(1-x_{i}\right) g_{i}(x)\right)$. The following property states the equivalence of $Q 01$ and our positive compact linearization $R L_{\text {comp }}$ :

Proposition 1.1. Problems $Q 01$ and $R L_{\text {comp }}$ are equivalent in the sense that, from any optimal solution of the one, we can build a solution to the other, with the same objective value.

Program $R L_{\text {comp }}$ has the $n$ variables $x_{i}$ and the $m+p$ constraints of the initial program $Q 01$. In addition, it has $2 n$ nonnegative variables $h_{i}$ and $h_{i}^{\prime}$, and $2 n$ additional constraints.

Other linear compact reformulations are reported in literature. Most of them are inspired from [14]. Our positive compact linearization can also be considered as a variation of [14] and has the advantage of working on a first reformulation of the objective function as a constant $c$ plus a quadratic function, nonnegative over $\bar{X}$. Hence, we can be sure that the lower bound computed by continuous relaxation of $R L_{\text {comp }}$ is at least $c$. Observe also that this last property holds for any correctly chosen values of the upper bounds $\bar{f}_{i}$ et $\bar{g}_{i}$ on functions $f_{i}(x)$ and $g_{i}(x)$.

\subsection{Quadratic COnVEX Reformulation [6]}

A quadratic convex reformulation of $Q 01$ consists in finding a quadratic convex function $F_{c}(x)$ that satisfies $F(x)=F_{c}(x)$ for any feasible solution $x$ in $X$. Hence, 
the following problem $R Q_{\text {conv }}$ is equivalent to $Q 01$ :

$$
\begin{aligned}
& R Q_{\text {conv }}: \operatorname{Min} F_{c}(x)=q_{0}^{\prime}+\sum_{i=1}^{n} q_{i}^{\prime} x_{i}+\sum_{i=1}^{n} \sum_{j=1}^{n} q_{i j}^{\prime} x_{i} x_{j} \\
& \text { s.t. } \\
& \sum_{i=1}^{n} a_{k i} x_{i}=b_{k} \quad k=1, \ldots, m \\
& \sum_{i=1}^{n} a_{\ell i}^{\prime} x_{i} \leq b_{\ell}^{\prime} \quad \ell=1, \ldots, p \\
& x \in\{0,1\}^{n} .
\end{aligned}
$$

Function $F_{c}$ is convex if and only if its Hessian matrix is positive semidefinite.

Such reformulation of $Q 01$ is always possible. One can for example use equality $x_{i}^{2}=x_{i}$ satisfied by any binary variable $x_{i}$ in order to raise up the diagonal coefficients of matrix $Q=\left(q_{i j}\right)$ by a large-enough constant. This diagonal perturbation can then immediately be balanced by the coefficients of the linear terms $q_{i}$. Based on this idea, a simple convex reformulation was already proposed in [17] and consists in a perturbation of the diagonal terms of $Q$ by its smallest eigenvalue. For our example $E$, the smallest eigenvalue of $Q$ equals -56.88 and $\phi(x)$ can be reformulated as $\phi_{c}(x)=\phi(x)+56.88 \sum_{i=1}^{5}\left(x_{i}^{2}-x_{i}\right)$ and the minimum of $\phi_{c}$ over $\bar{X}$ is equal to -119.31 .

Problem $R Q_{\text {conv }}$ has precisely the same number of variables and constraints as problem $Q 01$.

\subsection{A COMPARISON CRITERION FOR REFORMULATIONS}

The linear and quadratic reformulations presented above have the following as a common property: solving their continuous relaxation is tractable in polynomial time, and is quick to compute. Indeed, the reformulated problem has, roughly speaking, the same size as the original problem $Q 01$. Hence a natural way to solve the mixed-01 reformulated problems is to submit them to standard solvers whose exact solution procedures are based on branch-and-bound and continuous relaxation. Further, we know that the efficiency of a branch-and-bound algorithm is strongly dependent upon the quality of the bound at the root of the branchand-bound tree, computed as the optimal value of the continuous relaxation of the reformulated problem. This provides us with a criteria for comparing reformulations. We consider that the quality of a reformulation is given by the tightness of its continuous relaxation. Within a given reformulation scheme, reformulation $F 1$ is better than reformulation $F 2$ if the continuous relaxation of $F 1$ leads to a tighter bound than the continuous relaxation of $F 2$. 


\section{BUILDing A COMPACT LINEAR REFORMULATION BY USE OF THE BOUND OF A LINEAR RELAXATION}

\subsection{The RLT-RELAXATiON}

The relaxation we use here in known in the literature under the name "RLTlevel 1" $[25,26]$. It consists in adding quadratic valid inequalities to $Q 01$ before a classical linearization step. The valid inequalities are obtained by multiplying every equality by $x_{i}$ and every inequality by $x_{i}$ and $\left(1-x_{i}\right)$. Then, each product of two variables $x_{i} x_{j}$ is replaced by a new real variable $y_{i j}$. Linearization constraints ensure the validity of the substitution. Finally, a continuous relaxation step provides the following linear program $P L_{p}$ that is precisely called the RLT-relaxation:

$$
\begin{aligned}
& P L_{p}: \text { Min } F(x, y)=\sum_{i=1}^{n} q_{i} x_{i}+\sum_{i=1}^{n} \sum_{j=1, j \neq i}^{n} q_{i j} y_{i j} \\
& \text { s.t. } \sum_{i=1}^{n} a_{k i} x_{i}=b_{k} \quad k=1, \ldots, m \\
& \sum_{i=1}^{n} a_{k i} y_{i j}=b_{k} x_{j} \quad k=1, \ldots, m ; j=1, \ldots, n \\
& y_{i j}=y_{j i} \quad i=1, \ldots, n ; j=1, \ldots, n: j \neq i \\
& y_{i i}=x_{i} \quad i=1, \ldots, n \\
& \sum_{i=1}^{n} a_{\ell i}^{\prime} x_{i} \leq b_{\ell}^{\prime} \quad \ell=1, \ldots, p \\
& \sum_{i=1}^{n} a_{\ell i}^{\prime} y_{i j} \leq b_{\ell}^{\prime} x_{j} \quad \ell=1, \ldots, p ; j=1, \ldots, n \\
& \sum_{i=1}^{n} a_{\ell i}^{\prime}\left(x_{i}-y_{i j}\right) \leq b_{\ell}^{\prime}\left(1-x_{j}\right) \quad \ell=1, \ldots, p ; j=1, \ldots, n \\
& y_{i j} \leq x_{i} \quad i=1, \ldots, n ; j=1, \ldots, n, j \neq i \\
& x_{i}+x_{j}-y_{i j} \leq 1 \quad i=1, \ldots, n-1 ; j=i+1, \ldots, n \\
& x_{i} \leq 1 \quad i=1, \ldots, n \\
& x_{i} \geq 0 \quad i=1, \ldots, n \\
& y_{i j} \geq 0 \quad i=1, \ldots, n ; j=1, \ldots, n, j \neq i .
\end{aligned}
$$

This relaxation can be viewed as a strengthening trick for the classical linearization recalled in the Introduction. The bound computed by solving problem $P L_{p}$ is 
known to be quite efficient but rather time consuming because of the important size of $P L_{p}$. This makes these bounds difficult to incorporate into a branch-andbound algorithm.

Note that other 'lift-and-project' techniques are presented in the literature such as $[2,3,22,25]$.

\subsection{Using a dual SOlution of $P L_{p}$ In ORder to BUild a Positive Linear COMPACT REFORMULATION}

The following proposition shows how a reformulation of the objective function $F(x)$, in the form (1), can be obtained from any dual solution of the RLTrelaxation.

Proposition 2.1. For any dual feasible solution of $P L_{p}$, having objective value $V$, there exists $L(x), f_{i}(x)$, and $g_{i}(x)$ such that,

$\forall x \in X$

$$
F(x)=V+L(x)+\sum_{i=1}^{n} x_{i} f_{i}(x)+\sum_{i=1}^{n}\left(1-x_{i}\right) g_{i}(x)
$$

where $L(x), f_{i}(x)$, and $g_{i}(x)$ are linear functions, nonnegative over the relaxed set $\bar{X}$.

Proof. (Use the Property in Appendix A) Let $s_{i}$ (resp. $s_{i j}$ ) be the slack variables of the inequalities in the dual of $P L_{p}$ associated to $x_{i}$ (resp. $y_{i j}$ ). Given a dual feasible solution of $P L_{p}$, having objective value $V$, we take $\tilde{s}_{i}$ and $\tilde{s}_{i j}$, values of variables $s_{i}$ and $s_{i j}$, and the dual variables values $\tilde{v}^{1}$ (resp. $\left.\tilde{v}^{2}, \tilde{v}^{3}, \tilde{v}^{4}, \tilde{v}^{5}, \tilde{v}^{6}\right)$ associated to inequalities (2) (resp. (3), (4), (5), (6), (7)). We get,

For any feasible solution $(x, y)$ of $P L_{p}$ :

$$
\begin{aligned}
F(x, y)= & V+\sum_{i=1}^{n} \tilde{s}_{i} x_{i}+\sum_{i=1}^{n} \sum_{j=1, j \neq i}^{n} \tilde{s}_{i j} y_{i j}+\sum_{\ell=1}^{p} \tilde{v}_{\ell}^{1}\left(b_{\ell}^{\prime}-\sum_{i=1}^{n} a_{\ell i}^{\prime} x_{i}\right) \\
& +\sum_{\ell=1}^{p} \sum_{j=1}^{n} \tilde{v}_{\ell j}^{2}\left(b_{\ell}^{\prime} x_{j}-\sum_{i=1}^{n} a_{\ell i}^{\prime} y_{i j}\right)+\sum_{\ell=1}^{p} \sum_{j=1}^{n} \tilde{v}_{\ell j}^{3}\left(b_{\ell}^{\prime}\left(1-x_{j}\right)\right. \\
& \left.-\sum_{i=1}^{n} a_{\ell i}^{\prime}\left(x_{i}-y_{i j}\right)\right)+\sum_{i=1}^{n} \sum_{j=1, j \neq i}^{n} \tilde{v}_{i j}^{4}\left(x_{i}-y_{i j}\right) \\
& +\sum_{i=1}^{n-1} \sum_{j=i+1}^{n} \tilde{v}_{i j}^{5}\left(1+y_{i j}-x_{i}-x_{j}\right)+\sum_{i=1}^{n} \tilde{v}_{i}^{6}\left(1-x_{i}\right) .
\end{aligned}
$$


For any $x \in X$, there exists a unique $y$ such that $(x, y)$ is a feasible solution to $P L_{p}$. This $y$ is defined as $y_{i j}=x_{i} x_{j}$, and gives, for any $x \in X$ :

$$
\begin{aligned}
F(x)= & V+\sum_{i=1}^{n} \tilde{s}_{i} x_{i}+\sum_{i=1}^{n} \sum_{j=1, j \neq i}^{n} \tilde{s}_{i j} x_{i} x_{j}+\sum_{\ell=1}^{p} \tilde{v}_{\ell}^{1}\left(b_{\ell}^{\prime}-\sum_{i=1}^{n} a_{\ell i}^{\prime} x_{i}\right) \\
& +\sum_{\ell=1}^{p} \sum_{j=1}^{n} \tilde{v}_{\ell j}^{2} x_{j}\left(b_{\ell}^{\prime}-\sum_{i=1}^{n} a_{\ell i}^{\prime} x_{i}\right)+\sum_{\ell=1}^{p} \sum_{j=1}^{n} \tilde{v}_{\ell j}^{3}\left(1-x_{j}\right)\left(b_{\ell}^{\prime}-\sum_{i=1}^{n} a_{\ell i}^{\prime} x_{i}\right) \\
& +\sum_{i=1}^{n} \sum_{j=1, j \neq i}^{n} \tilde{v}_{i j}^{4} x_{i}\left(1-x_{j}\right)+\sum_{i=1}^{n-1} \sum_{j=i+1}^{n} \tilde{v}_{i j}^{5}\left(1-x_{i}\right)\left(1-x_{j}\right)+\sum_{i=1}^{n} \tilde{v}_{i}^{6}\left(1-x_{i}\right) .
\end{aligned}
$$

Let:

$$
\begin{aligned}
L(x) & =\sum_{i=1}^{n} \tilde{s}_{i} x_{i}+\sum_{i=1}^{n} \tilde{v}_{i}^{6}\left(1-x_{i}\right)+\sum_{\ell=1}^{p} \tilde{v}_{\ell}^{1}\left(b_{\ell}^{\prime}-\sum_{i=1}^{n} a_{\ell i}^{\prime} x_{i}\right) \\
f_{i}(x) & =\sum_{j=1, j \neq i}^{n} \tilde{s}_{i j} x_{j}+\sum_{j=1, j \neq i}^{n} \tilde{v}_{i j}^{4}\left(1-x_{j}\right)+\sum_{\ell=1}^{p} \tilde{v}_{\ell i}^{2}\left(b_{\ell}^{\prime}-\sum_{j=1}^{n} a_{\ell j}^{\prime} x_{j}\right) \\
g_{i}(x) & =\sum_{j=i+1}^{n} \tilde{v}_{i j}^{5}\left(1-x_{j}\right)+\sum_{\ell=1}^{p} \tilde{v}_{\ell i}^{3}\left(b_{\ell}^{\prime}-\sum_{j=1}^{n} a_{\ell j}^{\prime} x_{j}\right)
\end{aligned}
$$

observing that, for any $x \in \bar{X}$, the above functions are nonnegative, we obtain the reformulation of $F(x)$ as (1), i.e.:

$$
\forall x \in X, \quad F(x)=V+L(x)+\sum_{i=1}^{n} x_{i} f_{i}(x)+\sum_{i=1}^{n}\left(1-x_{i}\right) g_{i}(x) .
$$

Finally, we determine the upper bounds: $\bar{f}_{i}=\operatorname{Max}\left\{f_{i}(x): x \in X\right\}$ and $\bar{g}_{i}=$ $\operatorname{Max}\left\{g_{i}(x): x \in X\right\}$ in order to get a positive linear compact reformulation associated to the given dual solution of $P L_{p}$.

Corollary 2.2. For any dual solution of $P L_{p}$ having objective value $V$, we can build a positive linear compact reformulation which continuous relaxation value is at least $V$. The best value of $V$ is obviously obtained from an optimal solution.

Let us observe that Adams et al. [1] build a different compact linearization, more directly inspired from [14] and whose continuous relaxation is equal to the optimal value of $P L_{p}$. An additional advantage of Corollary 2.2 is that it allows to reformulate the objective function $F$ as the optimal value plus a nonnegative function over $\bar{X}$. This reformulation can be used for other exact or approximate solution approaches. 
Example. Let us reformulate the objective function $\phi(x)$ of $E$ once the RLTrelaxation has been solved:

$$
\begin{aligned}
\phi(x)= & -67.52 \\
& +x_{1}\left(109.83 x_{4}+30.07 x_{5}\right) \\
& +x_{2}\left(129.90 x_{4}+3.52\left(1-x_{3}\right)\right) \\
& +x_{3}\left(1.26\left[-2-\left(-x_{1}+2 x_{2}-5 x_{3}-2 x_{4}+2 x_{5}\right)\right]\right) \\
& +x_{4}\left(6.95\left[-2-\left(-x_{1}+2 x_{2}-5 x_{3}-2 x_{4}+2 x_{5}\right)\right]\right) \\
& +x_{5}\left(52.34\left(1-x_{3}\right)+11.22\left[-2-\left(-x_{1}+2 x_{2}-5 x_{3}-2 x_{4}+2 x_{5}\right)\right]\right) \\
& +\left(1-x_{2}\right)\left(0.74\left[-2-\left(-x_{1}+2 x_{2}-5 x_{3}-2 x_{4}+2 x_{5}\right)\right]\right) .
\end{aligned}
$$

We can now define functions $f_{i}(x)$ and $g_{i}(x)$,

$$
\begin{aligned}
& f_{1}(x)=109.83 x_{4}+30.07 x_{5} \\
& f_{2}(x)=129.90 x_{4}+3.52\left(1-x_{3}\right) \\
& f_{3}(x)=1.26\left(-2-\left(-x_{1}+2 x_{2}-5 x_{3}-2 x_{4}+2 x_{5}\right)\right) \\
& f_{4}(x)=6.95\left(-2-\left(-x_{1}+2 x_{2}-5 x_{3}-2 x_{4}+2 x_{5}\right)\right) \\
& f_{5}(x)=52.34\left(1-x_{3}\right)+11.22\left(-2-\left(-x_{1}+2 x_{2}-5 x_{3}-2 x_{4}+2 x_{5}\right)\right) \\
& g_{2}(x)=0.74\left(-2-\left(-x_{1}+2 x_{2}-5 x_{3}-2 x_{4}+2 x_{5}\right)\right)
\end{aligned}
$$

compute the upper bounds,

$\bar{f}_{1}=139.90, \bar{f}_{2}=133.42, \bar{f}_{3}=7.56, \bar{f}_{4}=41.7, \bar{f}_{5}=67.32, \bar{g}_{2}=4.44$.

and finally, build the following program $R L_{\text {comp }}$ :

$$
\begin{aligned}
R L_{\text {comp }_{E}}: \operatorname{Min} & \phi(x)=-67.52+\sum_{i=1}^{5} h_{i}+h_{2}^{\prime} \\
\text { s.t. } & \\
& -x_{1}+2 x_{2}-5 x_{3}-2 x_{4}+2 x_{5} \leq-2 \\
& x_{1}+x_{2}+x_{4}+x_{5}=2 \\
& h_{i} \geq f_{i}(x)-\bar{f}_{i}\left(1-x_{i}\right) \quad i=1, \ldots, 5 \\
& h_{2}^{\prime} \geq g_{2}(x)-\bar{g}_{2} x_{2} \\
& x \in\{0,1\}^{n} \quad i=1, \ldots, 5 \\
& h_{i} \geq 0 \\
& h_{2}^{\prime} \geq 0
\end{aligned}
$$

The optimal value of the continuous relaxation of $R L_{\text {comp }_{E}}$ is -67.52 .

In Appendix B we apply the linear compact reformulation of Glover [14] to the initial problem $E$. Its continuous relaxation value is -106.84 that is significantly lower than the continuous relaxation value of our positive linear compact reformulation. 


\section{BUILDing A QUADRATIC CONVEX REFORMULATION BY USE OF THE BOUND OF A SEMIDEFINITE RELAXATION}

In this section, we recall the Quadratic Convex Reformulation (QCR) method introduced in [6]. As we will see, a parallel can be made with the linearization approach presented in Section 2.

\subsection{A SEmidefinite Relaxation of $Q 01$}

Let us consider again problem Q01. We multiply every equality constraint $\sum_{i=1}^{n} a_{k i} x_{i}=b_{k}$ by $x_{i}$ and then replace the products $x_{i} x_{j}$ by variables $X_{i j}$, we obtain the following equivalent problem:

$$
\begin{aligned}
& \operatorname{Min} F(x)=\sum_{i=1}^{n} q_{i} x_{i}+\sum_{i=1}^{n} \sum_{j=i, j \neq i}^{n} q_{i j} X_{i j} \\
& \text { s.t. } \\
& \sum_{i=1}^{n} a_{k i} x_{i}=b_{k} \quad k=1, \ldots, m \\
& \sum_{i=1}^{i=1} a_{k i} X_{i j}=b_{k} x_{j} k=1, \ldots, m ; j=1, \ldots, n \\
& \sum_{i=1}^{n} a_{\ell i}^{\prime} x_{i} \leq b_{\ell}^{\prime} \quad \ell=1, \ldots, p \\
& \stackrel{X}{i j}_{i j}=x_{i} x_{j} \quad i=1, \ldots, n ; j=1, \ldots, n \text {. } \\
& x \in\{0,1\}^{n}
\end{aligned}
$$

The semidefinite relaxation consists in replacing the set of constraints $X_{i j}=x_{i} x_{j}$ with the linear matrix inequality $X-x x^{t} \succeq 0$. By Schur's Lemma, $X-x x^{t} \succeq 0$ is equivalent to $\left(\begin{array}{ll}1 & x^{t} \\ x & X\end{array}\right) \succeq 0$.

The obtained SDP relaxation is the following:

$$
\begin{aligned}
& S D P: \operatorname{Min} \sum_{i=1}^{n} q_{i} x_{i}+\sum_{i=1}^{n} \sum_{j=i, j \neq i}^{n} q_{i j} X_{i j} \\
& \text { s.t. } \sum_{i=1}^{n} a_{k i} x_{i}=b_{k} \quad k=1, \ldots, m \\
& \sum_{j=1}^{n} a_{k j} X_{i j}=b_{k} x_{j} \quad k=1, \ldots, m ; j=1, \ldots, n \\
& \sum_{i=1}^{n} a_{\ell i}^{\prime} x_{i} \leq b_{\ell}^{\prime} \quad \ell=1, \ldots, p \\
& X_{i i}=x_{i} \quad i=1, \ldots, n \\
& \left(\begin{array}{ll}
1 & x^{t} \\
x & X
\end{array}\right) \succeq 0 \quad x \in \mathbb{R}^{n}, X \in S_{n}
\end{aligned}
$$


where $S_{n}$ is the set of $n \times n$ symmetric matrices.

\subsection{USING AN OPTIMAL SOLUTION TO $S D P$ IN ORDER TO BUILD A QUADRATIC REFORMULATION}

The QCR method consists in reformulating problem $Q 01$ by adding a combination of quadratic functions that vanish on the feasible solution set $X$. For any $\alpha \in \mathbb{R}^{m \times n}$ and $u \in \mathbb{R}^{n}$, let us consider the following quadratic function:

$$
\begin{array}{r}
F_{\alpha, u}(x)=\sum_{i=1}^{n} q_{i} x_{i}+\sum_{i=1}^{n-1} \sum_{j=i, j \neq i}^{n} q_{i j} x_{i} x_{j}+\sum_{k=1}^{m}\left(\sum_{i=1}^{n} \alpha_{k i} x_{i}\right)\left(\sum_{j=1}^{n} a_{k j} x_{j}-b_{k}\right) \\
+\sum_{i=1}^{n} u_{i}\left(x_{i}^{2}-x_{i}\right) .
\end{array}
$$

Function $F_{\alpha, u}$ is a reformulation of $F$ since for all $x \in X, F_{\alpha, u}(x)$ is equal to $F(x)$. We focus our interest on reformulations of $F$ by $F_{\alpha, u}$ where $F_{\alpha, u}(x)$ is convex over $\mathbb{R}^{n}$. Once $F(x)$ is transformed into a convex function, the reformulated problem can be solved by mixed integer convex quadratic programming. Our objective now is to find values for parameters $\alpha \in \mathbb{R}^{m \times n}$ and $u \in \mathbb{R}^{n}$ such that $F_{\alpha, u}(x)$ is convex on the one hand and the optimal value of the continuous relaxation of the reformulated problem is maximized on the other hand. It was proved in [6] that solving the above semidefinite relaxation $S D P$ allows to deduce optimal values for parameters $\alpha$ and $u$. More precisely, the optimal values $u_{i}^{*}$ of $u_{i}(i=1, \ldots, n)$ are given by the optimal values of the dual variables associated with constraints $(9)$ and the optimal values $\alpha_{i k}^{*}$ of $\alpha_{i k}(i=1, \ldots, n ; k=1, \ldots, m)$ are given by the optimal values of the dual variables associated to constraints (8).

The resulting quadratic convex reformulation is then:

$$
\begin{aligned}
& R Q_{\text {conv }}: \operatorname{Min} F_{\alpha^{*}, u^{*}}(x) \\
& \text { s.t. } \\
& \sum_{i=1}^{n} a_{k i} x_{i}=b_{k} \quad k=1, \ldots, m \\
& \sum_{i=1}^{n} a_{\ell i}^{\prime} x_{i} \leq b_{\ell}^{\prime} \quad \ell=1, \ldots, p . \\
& x \in\{0,1\}^{n}
\end{aligned}
$$

It is proved in [6] that $v_{S D P}$, the optimal value to $S D P$ is equal to the optimal value of the continuous relaxation of $R Q_{\text {conv }}$.

Remark 3.1. In a similar way as in the linear reformulation, we reformulate the objective function $F(x)$ as a constant plus a convex quadratic function that is nonnegative for any feasible solution $x \in \bar{X}$. Indeed, let $g(x)=F_{\alpha^{*}, u^{*}}(x)-v_{S D P}$, we have $F(x)=v_{S D P}+g(x)$ and $g(x) \geq 0 \forall x \in \bar{X}$. 
Example. The SDP relaxation of our example $E$ is:

$$
\begin{array}{rlr}
S D P_{E}: \text { Min } & -9 x_{1}-7 x_{2}+2 x_{3}+23 x_{4}+12 x_{5}-48 X_{12}+4 X_{13}+36 X_{14} & \\
& -24 X_{15}-7 X_{23}+36 X_{24}-84 X_{25}+40 X_{34}+4 X_{35}-88 X_{45} & \\
\text { s.t. } & & \\
& x_{1}-2 x_{2}+5 x_{3}+2 x_{4}-2 x_{5} \geq 2 & \\
& x_{1}+x_{2}+x_{4}+x_{5}=2 & \\
& X_{12}+X_{14}+X_{15}=x_{1} & \leftarrow \alpha_{2}^{*} \\
& X_{12}+X_{24}+X_{25}=x_{2} & \leftarrow \alpha_{3}^{*} \\
& X_{13}+X_{23}+X_{34}+X_{35}=2 x_{3} & \leftarrow \alpha_{4}^{*} \\
& X_{14}+X_{24}+X_{45}=x_{4} & \leftarrow \alpha_{5}^{*} \\
& X_{15}+X_{25}+X_{45}=x_{5} & \leftarrow u_{1}^{*} \\
& X_{11}=x_{1} & \leftarrow u_{2}^{*} \\
& X_{22}=x_{2} & \leftarrow u_{3}^{*} \\
& X_{33}=x_{3} & \leftarrow u_{4}^{*} \\
& X_{44}=x_{4} & \leftarrow u_{5}^{*} \\
& X_{55}=x_{5} & \\
& \left(\begin{array}{l}
1 x^{t} \\
x X^{*}
\end{array}\right) .0 . & \\
& x \in \mathbb{R}^{5}, X \in S_{5} &
\end{array}
$$

The optimal solution value of $S D P_{E}$ equals -81.38. It is also the optimal solution value of the continuous relaxation of the reformulated problem $R Q_{\text {conv }_{E}}$. Parameters $u^{*}$ and $\alpha^{*}$ that allow to build $R Q_{\text {conv }_{E}}$ are obtained from the optimal solution of $S D P_{E}$ :

$$
\begin{aligned}
R Q_{\text {conv }_{E}}: \min & \phi(x)+\left(11.2 x_{1}+26.3 x_{2}-15 x_{3}-21.8 x_{4}+42.32 x_{5}\right) \\
& \left(x_{1}+x_{2}+x_{4}+x_{5}-2\right)+17.24\left(x_{1}^{2}-x_{1}\right)+0.34\left(x_{2}^{2}-x_{2}\right) \\
& +24.9\left(x_{3}^{2}-x_{3}\right)+132.74\left(x_{4}^{2}-x_{4}\right)-15.38\left(x_{5}^{2}-x_{5}\right) \\
\text { s.t. } & \\
& x_{1}-2 x_{2}+5 x_{3}+2 x_{4}-2 x_{5} \geq 2 \\
& x_{1}+x_{2}+x_{4}+x_{5}=2 \\
& x \in\{0,1\}^{n} .
\end{aligned}
$$

\section{Conclusion}

Nowadays, efficient mathematical programming solvers are available to solve mixed-integer linear or convex quadratic problems. Consequently, try to use them for the general $0-1$ quadratic problem is attractive. For that, a preprocessing phase is necessary in order to reformulate the initial problem into a tractable form. The efficiency of this exact solution approach strongly depends on the quality of the bound given by the continuous relaxation of the reformulation and also on the size of this reformulation. For both classes of solvers - mixed-integer linear or 
mixed-integer convex quadratic - we have proposed a concise reformulation technique that incorporates the optimal values of tight linear or positive semidefinite relaxations. A first computational comparison of the quadratic versus the linear approach was carried out for a task allocation problem and is reported in [10]. Other experimental results considering one of the two approaches are presented in $[1,5-7]$ and show the efficiency of these methods. Future research topics include obtaining reformulations based on even tighter relaxations.

\section{APPENDiX A}

Let $\mathrm{P}$ be the following linear program:

$$
\begin{array}{ll}
\text { P : Min } & f(x)=\sum_{i=1}^{n} c_{i} x_{i} \\
\text { s.t. } & \\
& \sum_{i=1}^{n} a_{k i} x_{i}=b_{k} \quad k=1, \ldots, m \\
& \sum_{i=1}^{n} a_{\ell i}^{\prime} x_{i} \leq b_{\ell}^{\prime} \quad \ell=1, \ldots, p \\
x_{i} \geq 0 & i=1, \ldots, n
\end{array}
$$

and let $\mathrm{D}$ be its dual problem :

$$
\begin{aligned}
& \mathrm{D}: \operatorname{Max} g(u, v)=\sum_{k=1}^{m}\left(-b_{k}\right) u_{k}+\sum_{\ell=1}^{p}\left(-b_{\ell}^{\prime}\right) v_{\ell} \\
& \text { s.t. } \\
& \sum_{k=1}^{m}\left(-a_{k i}\right) u_{k}+\sum_{\ell=1}^{p}\left(-a_{\ell i}^{\prime}\right) v_{\ell} \leq c_{i} \quad i=1, \ldots, n . \\
& u_{k} \in R ; v_{\ell} \geq 0
\end{aligned}
$$

Proposition. Let $s_{i}, i=1, \ldots, n$ be the non-negative slack variables associated to constraints $(10)$ and let $(\tilde{u}, \tilde{v}, \tilde{s})$ be a feasible solution for the dual problem $\mathrm{D}$. Then, for any feasible solution $x$ of the primal $\mathrm{P}$, we have:

$$
f(x)=g(\tilde{u}, \tilde{v})+\sum_{\ell=1}^{p} \tilde{v}_{\ell}\left(b_{\ell}^{\prime}-\sum_{i=1}^{n} a_{\ell i}^{\prime} x_{i}\right)+\sum_{i=1}^{n} \tilde{s}_{i} x_{i} .
$$

Proof. By definition of the slack variables $s_{i}: c_{i}=\sum_{k=1}^{m}\left(-a_{k i}\right) \tilde{u}_{k}+\sum_{\ell=1}^{p}\left(-a_{\ell i}^{\prime}\right) \tilde{v}_{\ell}+\tilde{s}_{i}$.

We can now use the last identity in the expression of $f(x)$. 
We get, for any vector $x$ :

$$
\begin{aligned}
f(x)= & \sum_{i=1}^{n}\left(\sum_{k=1}^{m}\left(-a_{k i}\right) \tilde{u}_{k}+\sum_{\ell=1}^{p}\left(-a_{\ell i}^{\prime}\right) \tilde{v}_{\ell}+\tilde{s}_{i}\right) x_{i} \\
= & \sum_{k=1}^{m} \tilde{u}_{k}\left(-\sum_{i=1}^{n} a_{k i} x_{i}\right)+\sum_{\ell=1}^{p} \tilde{v}_{\ell}\left(-\sum_{i=1}^{n} a_{\ell i}^{\prime} x_{i}\right)+\sum_{i=1}^{n} \tilde{s}_{i} x_{i} \\
= & \sum_{k=1}^{m}\left(-b_{k}\right) \tilde{u}_{k}+\sum_{\ell=1}^{p}\left(-b_{\ell}^{\prime}\right) \tilde{v}_{\ell}+\sum_{k=1}^{m} \tilde{u}_{k}\left(b_{k}-\sum_{i=1}^{n} a_{k i} x_{i}\right) \\
& +\sum_{\ell=1}^{p} \tilde{v}_{\ell}\left(b_{\ell}^{\prime}-\sum_{i=1}^{n} a_{\ell i}^{\prime} x_{i}\right)+\sum_{i=1}^{n} \tilde{s}_{i} x_{i} \\
= & g(\tilde{u}, \tilde{v})+\sum_{k=1}^{m} \tilde{u}_{k}\left(b_{k}-\sum_{i=1}^{n} a_{k i} x_{i}\right)+\sum_{\ell=1}^{p} \tilde{v}_{\ell}\left(b_{\ell}^{\prime}-\sum_{i=1}^{n} a_{\ell i}^{\prime} x_{i}\right)+\sum_{i=1}^{n} \tilde{s}_{i} x_{i}
\end{aligned}
$$

now, for any feasible solution $x$ to $\mathrm{P}$, we have $b_{k}-\sum_{i=1}^{n} a_{k i} x_{i}=0$, and then:

$$
f(x)=g(\tilde{u}, \tilde{v})+\sum_{\ell=1}^{p} \tilde{v}_{\ell}\left(b_{\ell}^{\prime}-\sum_{i=1}^{n} a_{\ell i}^{\prime} x_{i}\right)+\sum_{i=1}^{n} \tilde{s}_{i} x_{i} .
$$

Observe that for any feasible solution $x, b_{k}^{\prime}-\sum_{i=1}^{n} a_{\ell i}^{\prime} x_{i} \geq 0, x_{i} \geq 0$ and coefficients $\tilde{v}_{\ell}$ et $\tilde{s}_{i}$ are $\geq 0$.

\section{Appendix B}

Recall our example $E$ presented all along the paper:

$$
\begin{array}{cl}
E: \operatorname{Min} & \phi(x)=-9 x_{1}-7 x_{2}+2 x_{3}+23 x_{4}+12 x_{5}-48 x_{1} x_{2}+4 x_{1} x_{3}+36 x_{1} x_{4} \\
& -24 x_{1} x_{5}-7 x_{2} x_{3}+36 x_{2} x_{4}-84 x_{2} x_{5}+40 x_{3} x_{4}+4 x_{3} x_{5}-88 x_{4} x_{5} \\
\text { s.t. } & \\
& x_{1}-2 x_{2}+5 x_{3}+2 x_{4}-2 x_{5} \geq 2 \\
& x_{1}+x_{2}+x_{4}+x_{5}=2 \\
& x_{1}, x_{2}, x_{3}, x_{4}, x_{5} \in\{0,1\} .
\end{array}
$$


Let us apply the classical linearization to it:

$$
\begin{aligned}
& \text { Min }-9 x_{1}-7 x_{2}+2 x_{3}+23 x_{4}+12 x_{5}-48 y_{12}+4 y_{13}+36 y_{14} \\
& -24 y_{15}-7 y_{23}+36 y_{24}-84 y_{25}+40 y_{34}+4 y_{35}-88 y_{45} \\
& \text { s.t. } \\
& -x_{1}+2 x_{2}-5 x_{3}-2 x_{4}+2 x_{5} \leq-2 \\
& x_{1}+x_{2}+x_{4}+x_{5}=2 \\
& y_{i j} \leq x_{i} \quad i<j ; q_{i j}<0 \\
& y_{i j} \leq x_{j} \quad i<j ; q_{i j}<0 \\
& 1-x_{i}-x_{j}+y_{i j} \geq 0 \quad i<j ; q_{i j}>0 \\
& y_{i j} \geq 0 \quad i<j ; q_{i j}>0 \\
& x_{1}, \ldots, x_{5} \in\{0,1\} \text {. }
\end{aligned}
$$

The optimal solution value equals -115 .

If one applies the compact linearization of Glover [14] to $E$ (see Sect. 1.1), we get the following linear program:

$$
\begin{array}{ll}
\text { Min } & -9 x_{1}-7 x_{2}+2 x_{3}+23 x_{4}+12 x_{5}+z_{1}+z_{2}+z_{3}+z_{4}+z_{5} \\
\text { s.t. } & \\
& -x_{1}+2 x_{2}-5 x_{3}-2 x_{4}+2 x_{5} \leq-2 \\
& x_{1}+x_{2}+x_{4}+x_{5}=2 \\
& z_{1} \geq-22 x_{1} \\
& z_{1} \geq-24 x_{2}+2 x_{3}+18 x_{4}-12 x_{5}-20\left(1-x_{1}\right) \\
& z_{2} \geq-69.5 x_{2} \\
& z_{2} \geq-24 x_{1}-3.5 x_{3}+18 x_{4}-42 x_{5}-14.5\left(1-x_{2}\right) \\
& z_{3} \geq-1.5 x_{3} \\
& z_{3} \geq 2 x_{1}-3.5 x_{2}+20 x_{4}+2 x_{5}-22\left(1-x_{3}\right) \\
& z_{4} \geq-24 x_{4} \\
& z_{4} \geq 18 x_{1}+18 x_{2}+20 x_{3}-44 x_{5}-56\left(1-x_{4}\right) \\
& z_{5} \geq-84 x_{5} \\
& z_{5} \geq-12 x_{1}-42 x_{2}+2 x_{3}-44 x_{4}+10\left(1-x_{5}\right) \\
& x_{1}, \ldots, x_{5} \in\{0,1\}
\end{array}
$$

which optimal value of the continuous relaxation equals -106.84 .

Below, we summarize the optimal values of different relaxations associated with example $E$ :

\begin{tabular}{|c|c|c|c|c|c|c|}
\hline $\begin{array}{c}\text { Opt. } \\
\text { value }\end{array}$ & $\begin{array}{c}\text { Classical } \\
\text { lin. }\end{array}$ & $\begin{array}{c}\text { Compact lin. } \\
\text { of Glover }\end{array}$ & RLT-1 & $\begin{array}{c}\text { Our positive } \\
\text { compact lin. }\end{array}$ & $\begin{array}{c}\text { Eigenvalue } \\
\text { reform. }\end{array}$ & $\begin{array}{c}\text { Our quad. } \\
\text { conv. reformulation }\end{array}$ \\
\hline-65 & -115 & -106.84 & -67.52 & -67.52 & -119.31 & -81.39 \\
\hline
\end{tabular}




\section{REFERENCES}

[1] W.P. Adams, R. Forrester and F. Glover, Comparisons and enhancement strategies for linearizing mixed 0-1 quadratic programs. Discrete Optim. 1(2) (2004) 99-120.

[2] W.P. Adams and H.D. Sherali, A tight linearization and an algorithm for 0-1 quadratic programming problems. Manage. Sci. 32 (1986) 1274-1290.

[3] W.P. Adams and H.D. Sherali, Mixed-integer bilinear programming problems. Math. Program. 59 (1993) 279-305.

[4] J.E. Beasley, Heuristic algorithms for the unconstrained binary quadratic programming problem. Technical report, Department of Mathematics, Imperial College of Science and Technology, London, England (1998).

[5] A. Billionnet and S. Elloumi, Using a mixed integer quadratic programming solver for the unconstrained quadratic 0-1 problem. Math. Program. 109 (2007) 55-68.

[6] A. Billionnet, S. Elloumi and M.C. Plateau, Improving the performance of standard solvers for quadratic 0-1 programs by a toight convex reformulation: the QCR method. Discrete Appl. Math., http://dx.doi.org/10.1016/j.dam.2007.007 (to appear).

[7] A. Billionnet, S. Elloumi and M.C. Plateau, Quadratic convex reformulation: a computational study of the graph bisection problem. Technical Report CEDRIC, http:// cedric.cnam.fr/PUBLIS/RC1003.pdf (2005).

[8] A. Billionnet and E. Soutif, Using a mixed integer programming tool for solving the 0-1 quadratic knapsack problem. INFORMS J. Comput. 16 (2004) 188-197.

[9] M.W. Carter, The indefinite zero-one quadratic problem. Discrete Appl. Math. 7 (1984) 23-44.

[10] S. Elloumi, Linear programming versus convex quadratic programming for the module allocation problem. Technical Report CEDRIC 1100, http://cedric.cnam.fr/ PUBLIS/RC1100.pdf (2005).

[11] R. Fortet, Applications de l'algèbre de boole en recherche opérationnelle. Rev. Fr. d'Automatique d'Informatique et de Recherche Opérationnelle 4 (1959) 5-36.

[12] R. Fortet, L'algèbre de boole et ses applications en recherche opérationnelle. Cahiers du Centre d'Etudes de Recherche Opérationnelle 4 (1960) 17-26.

[13] M. Garey and D. Johnson, Computers and intractibility: a guide to the theroy of np-completeness. W.H. freeman \& Co. (1979).

[14] F. Glover, Improved linear integer programming formulation of non linear integer problems. Manage. Sci. 22 (1975) 445-460.

[15] F. Glover, G.A. Kochenberger and B. Alidaee, Adaptative memory tabu search for binary quadratic programs. Manage. Sci. 44 (1998) 336-345.

[16] S. Gueye and P. Michelon, Miniaturized linearizations for quadratic 0/1 problems. Ann. Oper. Res. 140 (2005) 235-261.

[17] P.L. Hammer and A.A. Rubin, Some remarks on quadratic programming with 0-1 variables. RAIRO 3 (1970) 67-79.

[18] P.L. Hammer, P. Hansen and B. Simeone, Roof duality, complementation and persistency in quadratic 0-1 optimization. Math. Program. 28 (1984) 121-155.

[19] D.S. Johnson, C.R. Aragon, L.A. McGeoch and C. Schevon, Optimization by simulated annealing: an experimental evaluation; part1, graph partitioning. Oper. Res. 37 (1989) 865-892.

[20] B.W. Kernighan and S. Lin, An efficient heuristic procedure for partitioning graphs. The Bell System Technical Journal 49 (1970) 291-307.

[21] A. Lodi, K. Allemand and T.M. Liebling, An evolutionary heuristic for quadratic 0-1 programming. Eur. J. Oper. Res. 119 (1999) 662-670.

[22] L. Lovász and S. Schrijver, Cones of matrices and set-functions and 0-1 optimization. SIAM J. Optim. 1 (1991) 166-190.

[23] P. Merz and B. Freisleben, Greedy and local search heuristics for unconstrained quadratic programming. J. Heuristics 8 (2002) 197-213. 
[24] M.C. Plateau, A. Billionnet and S. Elloumi, Eigenvalue methods for linearly constrained quadratic 0-1 problems with application to the densest k-subgraph problem. In 6e congrès ROADEF, Tours, 14-16 février, Presses Universitaires Francois Rabelais, http://cedric.cnam.fr/PUBLIS/RC723.pdf (2005) 55-66.

[25] H.D. Sherali and W.P. Adams, A Reformulation-Linearization Technique for Solving Discrete and Continuous Nonconvex Problems. Kluwer Academic Publ., Norwell, MA (1999).

[26] H.D. Sherali and H. Tuncbilek, A reformulation-convexification approach for solving nonconvex quadratic programming problems. J. Glob. Optim. 7 (1995) 1-31. 